Louisiana State University

LSU Digital Commons

Faculty Publications

Department of Biological Sciences

$2-1-2016$

\title{
Next-generation sequencing-based 5 rapid amplification of cDNA ends for alternative promoters
}

\author{
Bambarendage P.U. Perera \\ Louisiana State University \\ Joomyeong Kim \\ Louisiana State University
}

Follow this and additional works at: https://digitalcommons.Isu.edu/biosci_pubs

\section{Recommended Citation}

Perera, B., \& Kim, J. (2016). Next-generation sequencing-based 5' rapid amplification of cDNA ends for alternative promoters. Analytical Biochemistry, 494, 82-84. https://doi.org/10.1016/j.ab.2015.11.006

This Article is brought to you for free and open access by the Department of Biological Sciences at LSU Digital Commons. It has been accepted for inclusion in Faculty Publications by an authorized administrator of LSU Digital Commons. For more information, please contact ir@lsu.edu. 
Notes \& tips

\title{
Next-generation sequencing-based $5^{\prime}$ rapid amplification of cDNA ends for alternative promoters
}

\author{
Bambarendage P.U. Perera, Joomyeong Kim* \\ Department of Biological Sciences, Louisiana State University, Baton Rouge, LA 70803, USA
}

\section{A R T I C L E I N F O}

\section{Article history:}

Received 26 October 2015

Received in revised form

3 November 2015

Accepted 9 November 2015

Available online 23 November 2015

\section{Keywords:}

5'RACE

NGS

Alternative promoters

Peg3

\begin{abstract}
A B S T R A C T
Mammalian genomes contain many unknown alternative first exons and promoters. Thus, we have modified the existing 5'RACE (5' rapid amplification of cDNA ends) approach into a next-generation sequencing (NGS)-based new protocol that can identify these alternative promoters. This protocol has incorporated two main ideas: (i) 5'RACE starting from the known second exons of genes and (ii) NGSbased sequencing of the subsequent cDNA products. This protocol also provides a bioinformatics strategy that processes the sequence reads from NGS runs. This protocol has successfully identified several alternative promoters for an imprinted gene, PEG3. Overall, this NGS-based 5'RACE protocol is a sensitive and reliable method for detecting low-abundant transcripts and promoters.
\end{abstract}

(c) 2015 Elsevier Inc. All rights reserved.
In eukaryotic cells, gene transcription is regulated through several cis-regulatory DNA elements such as promoters, enhancers, silencers, and insulators [1]. Identifying promoters is one of the initial steps for characterizing transcriptional regulation for any given gene. In model organisms, the promoters of the majority of genes have been identified through large-scale sequencing efforts [2]. According to recent results, however, transcripts are quite often detected from the upstream regions of many genes that are outside of the previously defined transcribed regions. This indicates that additional transcription start sites and alternative promoters may exist for many genes [3]. In the current study, thus, we have modified and adapted the existing $5^{\prime}$ RACE ( $5^{\prime}$ rapid amplification of cDNA ends $)^{1}$ [4] approach into a next-generation sequencing (NGS)-based new protocol that can identify the alternative promoters for these transcripts. The main strength of this new protocol is its sensitivity; this can detect the $5^{\prime}$ ends of mRNA that are of very low abundance. The main ideas and detailed steps for this protocol are described below.

Two main ideas have been incorporated into this new protocol. First, the initial cDNA for 5'RACE needs to be prepared with the

Abbreviations: 5'RACE, 5' rapid amplification of cDNA ends; cDNA, complementary DNA; NGS, next-generation sequencing; RT, reverse transcription; mRNA, messenger RNA; PCR, polymerase chain reaction; dGTP, deoxyguanosine triphosphate; TdT, terminal deoxynucleotidyl transferase; $P E G 3$, paternally expressed gene 3; UCSC, University of California, Santa Cruz.

* Corresponding author.

E-mail address: jkim@lsu.edu (J. Kim). reverse transcription (RT) reaction using gene-specific oligonucleotide primers (Fig. 1B). These gene-specific primers also need to be derived from the known second exons, but not from the known first exons, for genes. The known first exons are not a good choice because these exons do not have splicing acceptor sites at their $5^{\prime}$ ends. Instead, they have transcription start sites; thus, they cannot be connected to any upstream exons. By contrast, the known second exons have proper splicing acceptor sites; thus, they should be ideal regions for anchoring the upstream alternative exons that have not been identified so far. Second, potential unknown alternative first exons most likely represent the sequences that belong to very low-abundant transcripts given all of the sequencing efforts by the genomic community. Therefore, this new protocol uses NGSbased approaches to identify the sequences derived from these very minor transcripts. NGS-based sequencing should allow the identification of these low-abundant transcripts given their sequencing capacity (million reads per library). This new protocol, therefore, includes several steps that can easily convert polymerase chain reaction (PCR) products into NGS libraries.

The detailed steps for the new protocol are as follows (Fig. 1A; see also Supplementary Material 1 in the online supplementary material). In step 1, the total RNA isolated from tissue samples is reverse-transcribed with gene-specific primers (dark arrow in Fig. 1B). The subsequent cDNA needs to be purified through phenol/ chloroform extraction followed by ethanol precipitation. In step 2 , the cDNA is modified through the tailing reaction using deoxyguanosine triphosphate (dGTP) and terminal deoxynucleotidyl transferase (TdT). In step 3, a pool of the G-tailed cDNA is targeted 
A

$\underline{\text { Overall Scheme }}$

1. RT with gene-specific primers

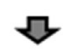

2. G-tailing of cDNA products

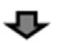

3. Nested PCR with internal primers

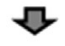

4. Ligation with barcoded adapters

5. NGS-based sequencing

6.

Sequence sorting

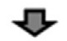

7. Map with the UCSC genome browser

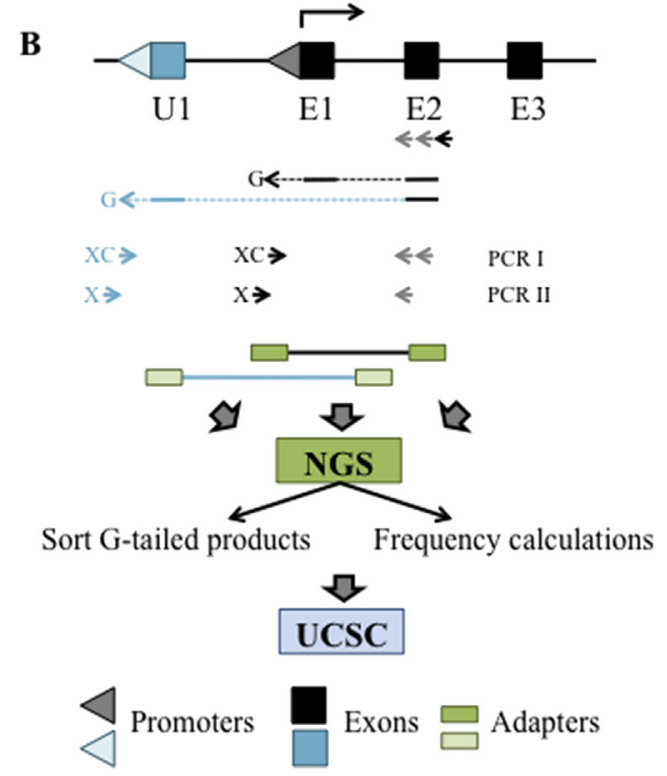

Fig.1. Overall scheme for the NGS-based 5'RACE protocol. A A flow chart shows the entire procedure of the NGS-based 5'RACE protocol. B A schematic illustration represents the genomic structure of a given locus, with its promoters and exons indicated by triangles and rectangles, respectively. The bent arrow indicates the transcriptional direction of the gene. The previously known transcript and its alternative transcript are shown in black and light blue, respectively. The total RNA isolated from the desired tissue is first reversetranscribed using gene-specific primers, as indicated by the black arrow underneath the exon structure. The resulting cDNA product is then purified and modified with the tailing reaction using dGTP and TdT, as indicated by the extended blue and black arrows with a G mark. The CDNA is then used as a template for a nested PCR I with the following two primers: an internal gene-specific primer (gray arrow) and a primer made of a unique sequence plus a C-tail (indicated by XC). The product from PCR I is subsequently used for PCR II with another internal gene-specific primer (gray arrow) and the second primer specific for the unique sequence (arrow indicated by X). The resulting products are end-repaired and ligated to barcoded adapters as shown by the green boxes. A set of several 5'RACE libraries can be pooled together for NGS-based sequencing. The sequences from NGS runs are sorted based on the barcoded adapters and also the G-tailed and gene-specific primer portion of the cDNA products. These sorted sequences are further divided into individual groups based on their different combinations of exon joining. These groups of the sorted sequences are analyzed to examine their exon structures using the UCSC genome browser. Each group with a different exon joining can also be analyzed for the frequency (or representation) in a given library. (For interpretation of the references to color in this figure legend, the reader is referred to the web version of this article.).

and enriched for a given gene through a nested PCR scheme involving two sets of primers: two gene-specific primers (gray arrows) and two primers targeting the G-tailed portion of cDNA (XC and X; blue arrows). A small amount of PCR products from each PCR needs to be separated on a $2 \%$ agarose gel to monitor the proper amplification of cDNA. In step 4, the amplified product from the second PCR is modified with the end repair reaction and later with the ligation reaction to be analyzed with NGS-based sequencing. The current protocol has been tested multiple times using an NGS platform (PGM2, Ion Torrent), which requires two specific sequences at either end of all the DNA fragments to be analyzed ( $A$ and $P$ adapters). Thus, the two adapters containing these sequences need to be ligated onto either end of the cDNA. An NGS platform usually allows the simultaneous sequencing of multiple libraries; thus, several cDNA libraries derived from multiple tissues are also ligated individually with a set of adapters with different barcodes. In step 5, the pool of individual barcoded libraries is amplified with PCR and subsequently sequenced using an NGS platform. In step 6, the raw sequence reads from NGS runs are sorted first by their barcodes and later by the sequences of the two primers: $\mathrm{X}$ and the gene-specific primer used for the second PCR of the nested scheme (Fig. 1B). This sorting process can be executed through several Unix command lines, which has been included as Supplementary Material 1 . These filtered reads are further processed to identify alternative exons using a PERL script, which can detect and count the number of raw reads that have different combinations of exon joining with the initial exon (E2 in Fig. 1B). In step 7, each group of the raw reads displaying different exon joining can be mapped to the genome sequence of a given species using the UCSC (University of California, Santa Cruz) genome browser, which will then identify new alternative exons. This bioinformatics pipeline along with one PERL script has been included as Supplementary Material 2.
The feasibility of this protocol has been tested through identifying alternative promoters for an imprinted gene called PEG3 (paternally expressed gene 3) (Fig. 2). This gene is composed of 9 exons that are spread throughout 25-kb genomic regions in both human and mouse, and it is highly expressed in the brain [5-7]. Interestingly, the 200-kb upstream region of this gene is well conserved among all of the mammals without any additional open reading frames (ORFs); thus, this region has been suspected to harbor other unknown cis-regulatory elements [8]. This new protocol, therefore, has been applied to test whether this region contains any unknown alternative promoters. First, the total RNA from mouse and human brains was individually used for generating 5'RACE cDNA libraries, and later these libraries were sequenced using an NGS platform. Each library derived on average several hundred thousand reads, and these raw reads were further analyzed with the bioinformatics strategy described previously. According to the results, the majority of cDNA sequences were indeed derived from the transcripts with the known exon combination (E1-E2): 93\% for mouse and 74\% for human PEG3 (Fig. 2B). However, this protocol also detected some minor transcripts from both mouse and human, and these transcripts were very low in abundance based on their representations in the libraries: $0.33 \%$ for mouse and $0.13 \%$ for human PEG3. Detailed inspection further revealed that these minor transcripts skip the known first exons but are connected to previously unknown alternative exons (U1 in Fig. 2A). It also revealed that the new U1 exons of PEG3 are not conserved between mouse and human genomic regions. Individual RT-PCR analyses indeed confirmed the presence of these minor transcripts for mouse and human PEG3 (Fig. 2C). Until now, there has never been any clue suggesting the presence of alternative promoters for the PEG3 locus, although this locus has been intensively studied for more than two decades. Thus, this new protocol 
A

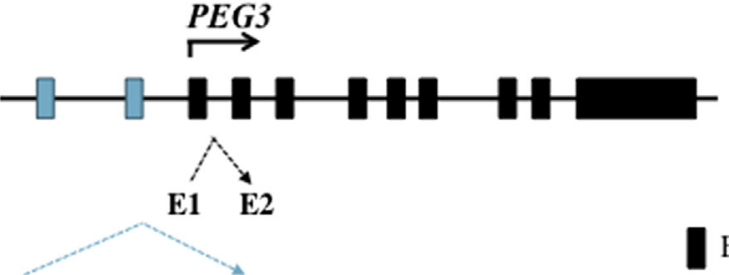

Exons

U1

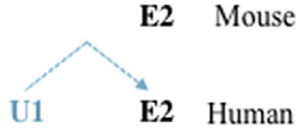

B

\begin{tabular}{|c|c|c|}
\hline Tissues & Mouse & Human \\
\hline No. of reads & 1,843 & 342,369 \\
\hline El(\%) & 93.54 & 74.65 \\
\hline mUl(\%) & $\mathbf{0 . 3 3}$ & N/A \\
\hline hUl(\%) & N/A & $\mathbf{0 . 1 3}$ \\
\hline
\end{tabular}

C

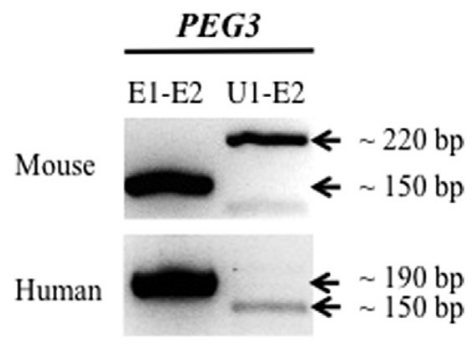

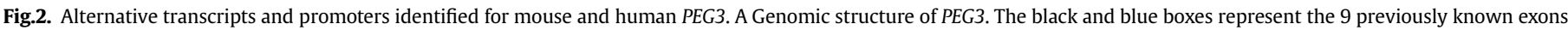

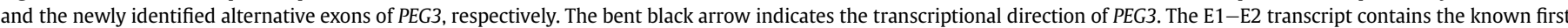

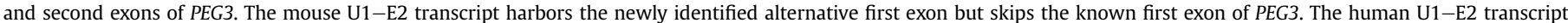

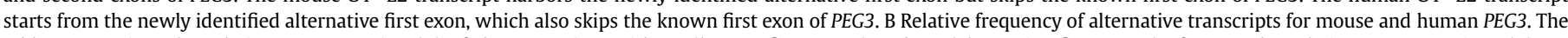

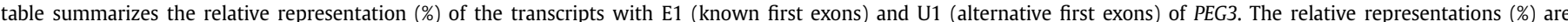

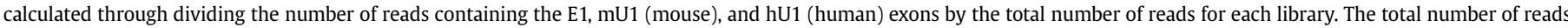

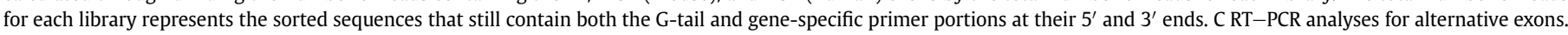

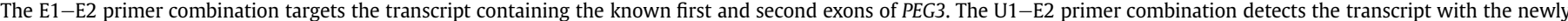

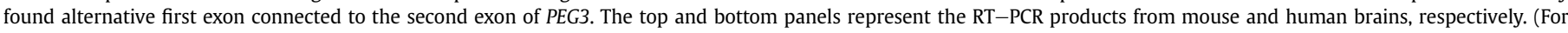
interpretation of the references to colour in this figure legend, the reader is referred to the web version of this article.)

appears to be very robust in identifying low-abundant transcripts and corresponding alternative promoters.

This newly developed protocol, NGS-based 5'RACE, may be used for other similar studies that intend to identify low-abundant transcripts for their gene of interest based on the following reasons. First, this protocol is very sensitive; it can detect very lowabundant transcripts and corresponding alternative promoters. As demonstrated above, this protocol successfully detected a minor transcript that makes up only $0.1 \%$ of all transcripts from human PEG3. This level of sensitivity cannot be achieved with the existing 5 'RACE protocols. This protocol also provides clear exon structures for a given locus, which is not always feasible with other NGS data, such as RNA-seq, due to their relatively short read lengths. Second, the new protocol can estimate the relative expression levels of the alternative transcripts for a given locus among various tissues or stages. The number of raw sequence reads belonging to each transcript can be easily converted into its relative representation in a given cDNA library, which can further indicate the strength of the corresponding alternative promoter. This type of information could be very important because the in vivo function of a given gene often manifests through its tissue and stage-specific alternative promoters. Third, this protocol is reliable and reproducible. We have tested more than 15 rounds of 5'RACE followed by NGS runs, and each round was consistently successful for identifying new alternative exons and promoters. Despite these strengths, however, the current protocol also has one caveat. We have been selecting DNA fragments less than $400 \mathrm{bp}$ in length for actual NGS runs due to the read length limitation that can be afforded by current NGS platforms. Thus, any 5'RACE cDNA products greater than $400 \mathrm{bp}$ in length cannot be sequenced through the current protocol. Nevertheless, this NGS-based 5'RACE protocol appears to be a very sensitive and reliable method for identifying low-abundant alternative transcripts and promoters.

\section{Acknowledgments}

We thank Drs. Scott Herke and Suman Lee for their help with NGS preparation and sequencing runs. We also thank the members of the JooKim lab for their discussion of the manuscript. This work was supported by the National Institutes of Health (R01-GM066225 and R01-GM097074).

\section{Appendix A. Supplementary data}

Supplementary data related to this article can be found at http:// dx.doi.org/10.1016/j.ab.2015.11.006.

\section{References}

[1] N.A. Campbell, J.B. Reece, L.A. Urry, M.L. Cain, S.A. Wasserman, P.V. Minorsky, R.B. Jackson, Biology, eighth ed., Pearson/Benjamin Cummings, Sydney, Australia, 2009, p. 150

[2] W.J. Kent, C.W. Sugnet, T.S. Furey, K.M. Roskin, T.H. Pringle, A.M. Zahler D. Haussler, The human genome browser at UCSC, Genome Res. 12 (2002) 996-1006.

[3] H. Sun, J. Wu, P. Wickramasinghe, S. Pal, R. Gupta, A. Bhattacharya, et al. Genome-wide mapping of RNA Pol-II promoter usage in mouse tissues by ChIPseq, Nucleic Acids Res. 39 (2011) 190-201.

[4] M.A. Frohman, M.K. Dush, G.R. Martin, Rapid production of full-length cDNAs from rare transcripts: amplification using a single gene-specific oligonucleotide primer, Proc. Natl. Acad. Sci. U. S. A. 85 (1988) 8998-9002.

[5] J. Kim, L. Ashworth, E. Branscomb, L. Stubbs, The human homolog of a mouseimprinted gene, Peg3, maps to a zinc finger gene-rich region of human chromosome 19q13.4, Genome Res. 7 (1997) 532-540.

[6] Y. Kuroiwa, T. Kaneko-Ishino, F. Kagitani, T. Kohda, L.L. Li, M. Tada, et al., Peg3 imprinted gene on proximal chromosome 7 encodes for a zinc finger protein, Nat. Genet. 12 (1996) 186-190.

[7] F. Relaix, X. Weng, G. Marazzi, E. Yang, N. Copeland, N. Jenkins, et al., Pw1, a novel zinc finger gene implicated in the myogenic and neuronal lineages, Dev. Biol. 177 (1996) 383-396.

[8] H. He, J. Kim, Regulation and function of the Peg3 imprinted domain, Genomics Inform. 12 (3) (2014) 105-113. 